\title{
Research on the Curent Situation of the Implementation of Extracurricular Sports Activities in Rural Middle Schools in Jiangxi Province -with Rural Middle Schools of Chongyi County as an Example
}

\author{
Renzhuo Zhang \\ School of Science, Jiangxi University of Science and Technology, Ganzhou, China \\ 47605591@qq.com
}

Keywords: Chongyi County; Rural middle school; Extracurricular sports activities; Current situationof implementation

\begin{abstract}
Through research methods such as document literature, questionnaire survey and data statistics, this article carried out researches on the current situationof extracurricular sports activities in rural middle schools of Chongyi County, Jiangxi Province, also, analysis on factors which would influence the implementation of extracurricular sports activities in rural middle school in Chongyi County, Jiangxi Province had been conducted, the results demonstrated that: factors such as lack of attention from school, poor organization and management of extracurricular sports activities, less school grounds and shorting equipment had influenced students' enthusiasm in participating in extracurricular sports activities. Focused on factors which would restrain development, this article proposed valuable and reasonable advice for reference of relevant parts.
\end{abstract}

\section{Introduction}

Extracurricular sports activities are activities carried out at spare time to keep fit, relieve pressure and relax both the body and the mind. They could strength students' physique, improve their body function and enrich their extracurricular life, play an important role in improving students' physique, athletic ability and the ability of making use of sports knowledge, increasing the quality of their study and life, as well as cultivating students' habits of exercising and the awareness of life-long exercise. In order to further learn about the current situationof extracurricular sports activities in rural middle schooland improve the implementation of those activities pointedly, this article carried out research with Chongyi County in Jiangxi Province as a topical case study. Chongyi County is within the original Central Soviet Area such as Gannan (the south of Jiangxi Province) whose rejuvenation and development are in counterpart support of State General Administration of Sports, also, most parts of the Chongyi County are rural areas, thus researches on the current situationof extracurricular sports activities in rural middle schools in Chongyi County could promote the physical education reform in rural middle schools of Jiangxi Province, and play an significant role in promoting the physical and mental health development of young students in Jiangxi Province, physical education reform in rural middle schools and shortening the distance between urban and rural schools.

\section{Current Situationof Extracurricular Sports Activities in Rural Middle Schools of Chongyi County}

The Attention Physical Education Teachers in Rural Middle Schools of Chongyi County Pay on Extracurricular Sports Activities. Through communication with parts of leaders and physical education teachers in rural middle schools of Chongyi County, it could be found that, instead of paying enough attention on the extracurricular sports activities, they just placed undue emphasis on the proportion of students entering schools of a higher level, ignored students' physical and mental development, the phenomenon of emphasizing on the classroom and neglecting extracurricular sports activities was quite serious. In order to increase enrollment rate, teachers in charge of the class and culture class teachers even occupied the time originally designed for extracurricular sports 
activities, which more seriously deprived the limited time for extracurricular sports activities. What's more, the physical education teachers' organization and management on extracurricular sports activities were poor, the physical education class was uninteresting, which led to loss of students' targets and motivation of sports, and reduction in enthusiasm, in the long run, the students would gradually loss interests in the physical education class, or even refuse to take part in activities.

Students' Recognition and Attitude on the Extracurricular Sports Activities. Most of the students in rural middle schools of Chongyi County did not have overall recognition on the extracurricular sports activities, while on the whole, their attitudes towards extracurricular sports activities were positive. The results were mainly caused by the environment and people around, the school and parents did not pay enough attention on activities, and the parents still regarded study as the main mission of students, and the extracurricular sports activities were dispensable, they could not delay study, thus they did neither understand nor support the extracurricular sports activities. Added with the lack of school grounds and equipments in rural middle schools, those who were not interested in activities would not participate in any more. Relatively, boys in the rural middle schools were more intended to take part in extracurricular sports activities than girls who were always in a passive condition, they thought that they could participate when the time allowed, and when the study was busy, they would give up, most of them held an indifferent attitude. More attention should be paid on those students, guiding them and strengthening their awareness of exercising.

\section{Motivation of Participating Extracurricular Sports Activities.}

Table 1 The results of survey on the motivation of participating in extracurricular sports activities of students in rural middle schools of Chongyi County (multiple choice)

\begin{tabular}{c|ccccc}
\hline Motivation & $\begin{array}{c}\text { Body } \\
\text { building }\end{array}$ & Interests & $\begin{array}{c}\text { Relieve study } \\
\text { pressure }\end{array}$ & Recreation & $\begin{array}{c}\text { Interpersonal } \\
\text { communication }\end{array}$ \\
\hline Boys & 79 & 63 & 49 & 38 & 14 \\
Girls & 62 & 58 & 47 & 33 & 11 \\
Total & 141 & 121 & 96 & 71 & 25 \\
Rank & 1 & 2 & 3 & 4 & 5 \\
\hline
\end{tabular}

It could be got from data in Table 1that: the primary motivation of students' participation in extracurricular sports activities was "body building", followed by "interests". This demonstrated that the motivation of most students were right and complied with the education purpose, this would benefit the physical and mental health development of them. While there were some students whose motivation did not comply with the direct education purpose of the school, the school should enhance education on them and tried to ensure that every student could realize the importance of extracurricular sports activities towards themselves.

Content of Extracurricular Sports Activities.

Table 2 The results of survey on the content of extracurricular sports activities in rural middle schools of Chongyi County (multiple choice)

\begin{tabular}{c|cccccc}
\hline $\begin{array}{c}\text { Content of } \\
\text { activities }\end{array}$ & $\begin{array}{c}\text { Track } \\
\text { and field }\end{array}$ & Basketball & Football & Badminton & Ping-pong & Others \\
\hline Boys & 13 & 59 & 12 & 27 & 46 & 17 \\
Girls & 9 & 15 & 3 & 81 & 21 & 13 \\
Total & 22 & 74 & 15 & 108 & 67 & 30 \\
Rank & 5 & 2 & 6 & 1 & 3 & 4 \\
\hline
\end{tabular}


It could be got from survey results in Table 2 that, the most frequently contacted sports events are basketball and ping-pong, and the content of extracurricular sports activities also concentrated on these two events, and followed by badminton. As known from students, this result was mostly caused by that there were only grounds and equipments for those events, most school even did not have decent filed for track and field and badminton, while the grounds for basketball and ping-pong table were in cement, rubber field were rare.

Students' Evaluation on School Grounds and Equipments. It could be seen from the results of questionnaire survey that, the school grounds and equipments in rural middle schools of Chongyi County were inadequate, and more than $90 \%$ of the students were dissatisfied with them, even some of the students were quite dissatisfied and considered that there were neither grounds nor equipments. This phenomenon was quite common in rural middle schools of Chongyi County. Small grounds and less equipment had become an important factor which influenced the physical education reform in rural middle schools of Chongyi County.

\section{Constraints on the Implementation of Extracurricular Sports Activities in Rural Middle Schools of Chongyi County}

Small Grounds and Less Equipments for Extracurricular Sports Activities. Enough campus grounds and equipment is an indispensable material condition for the implementation of extracurricular sports activities and exercises, and is also a priority of guaranteeing the physical education. The budget shortage had existed for ages, and this is also a direct cause of lack in school grounds and equipments. Though this condition has been improved in recent years, long term efforts are still needed to solve this problem. For lack of money, many schools had a small piece of concrete as the school ground, and some even did not have such concrete, the students could only exercise on a soil ground. And the so called equipments were only several basketballs, badminton rackets and skipping ropes, most of the physical education classes were free activities, they could not really exercise well, let alone developing good habits of exercise.

Constraints of Traditional Exam-oriented Education. Most students thought that the main reasons for not taking part in extracurricular sports activities were too much burden and pressure on study. The traditional exam-oriented concept had been deep-rooted in rural middle schools, and the phenomenon of paying unreasonably more attention on the classroom education was serious. In order to be enrolled in higher level school, the teachers in charge of the class and culture class teachers even occupied the time originally designed for extracurricular sports activities, which led to that students spent much more time in the classroom than outside. Therefore, the students even could not spare any time for sports and activities.

Occupation of Extracurricular Sports Times. Most of the students in rural middle schools in Chongyi County were non-resident students, and the resident students were relatively less, in most conditions, students should help to do some farm works after school, therefore, no more time would be left for extracurricular sports activities inside school. In addition, most leaders and teachers did not hold a supporting attitude on extracurricular sports activities, and were in lack of correct guidance on exercising, they paid too much attention on the education of cultural knowledge and ignored the fact that physical education was also an important factor of education.

Simple and Insufficient Content of Extracurricular Sports Activities. Existing extracurricular sports activities in rural middle schools were ball games, such as basketball and badminton, and other activities were rare, even the athletics which were simple and easy to organize were less. The simplification trend of activity content were serious now, which directly caused that the interests and demands of students could not be satisfied, and the loss of vitality and passion which they should originally hold for activities. 


\section{Measures on the Implementation of Extracurricular Sports Activities in Rural Middle Schools of Chongyi County}

More Attention on Extracurricular Sports Activities, and Get Rid of Constraints from Traditional Exam-oriented Education. Extracurricular sports activity could not only keep fit, but also could relieve study pressure and relax the body and mind, it is an indispensable part for the physical and mental development of students. Therefore, the educational departments and leaders should pay more attention on extracurricular sports activities, and tightly connect the implementation of extracurricular sports activities with the physical education reform in rural middle schools, and spend more efforts on carrying out extracurricular sports activities.

Relevant national educational departments should further enlarge investment on physical education in rural primary and middle schools, and add school grounds and facilities for them timely, and make sure that all funds are used on physical education and work the best. At the same time, supervise the extracurricular sports activities to ensure each student could exercise for one hour per day. On the problem of too much burden on enrollment, the leaders and physical education teachers should communicate with students'parents to inform them with the importance of extracurricular sports activities towards the healthy development of students, and pay more attention on extracurricular sports activities to get rid of the constraints of traditional exam-oriented education, do not force students to study cultural knowledge only through depriving the time for exercise. Then let the parents sincerely support extracurricular sports activities and thus promote the healthy development of students.

The School Should Guide actively, and Enrich the Content of Extracurricular Sports Activities to Realize the Diversity of Extracurricular Sports Activities. Currently, extracurricular sports activities are in a simplification trend which makes most students lose interests and develop the habit of unwilling to exercise, and thus the physical function decreases generally. Therefore, the school should prevent deterioration of such trend, formulate plans for extracurricular sports activities according to the principles of diversity and universality, then ensure the implementation of each extracurricular sports activity instead of activities just on the schedule, and various activities should be carried out to ensure the abundance of activities. The school should also actively guide students to do physical exercise at the same time of cultivating interests, and thus truly realize the diversity of extracurricular sports activities.

The School Should Learn about Students' Demand and Make Corresponding Plans to Increase Their Interest.Presently, extracurricular sports activities of rural middle schools are generally in low efficiency, though most schools have arranged extracurricular sports activities, most students could not finish with fulfilling both the quality and quantity requirements, most of them just finish in a perfunctory way, especially that, for rural middle schools, the time truly spent on extracurricular sports activities were less, the students even exclude exercise in the limited time, which makes the exercise efficiency even low. Therefore, the school should communicate with students timely to learn about their concrete need and the content of exercise they want, and then make corresponding plans for extracurricular sports activities, so as to increase students' interests and guarantee the effect of exercise and develop good habits.

\section{Conclusions}

Extracurricular sports activity is an important part of physical education, and the main means for students to participate in exercise and keep fit, it holds special educational meaning. While, in our country, the implementation of extracurricular sports activities was undesirable right now, especially in rural schools for constraints and influences of various factors, the implementation of extracurricular sports activities were unoptimistic. During the rapid development of social economy and deep reform of physical education, we should face the problem, and keep on proposing practical and reasonable suggestions and strategies, to better guarantee the implementation of extracurricular sports activities, and to let the young students truly realize the happiness of participating in those activities. 


\section{References}

[1] J.Li.B.C.Zhao and G.C.Liu, Review on the Current situationof Extracurricular sports activities in Rural Schools in Our Country. Guizhou Sports Science and Technology. (2009) No. 3. (In Chinese)

[2] M.Zhang, Research and Analysis on the Current situationof Extracurricular Sports Activities in Rural Middle Schools-with Leshan Area as an Example. Time Education. (2007) No. 29. (In Chinese)

[3] Research and Analysis on the Current situationof School Grounds in Rural Middle Schools in Jiangxi Province. The Science Education Article Collects (the late magazine). (2007) No. 12. (In Chinese)

[4] S.K.Ma, The Importance of Sports towards Modern Health. Youth Literator. (2009) No. 16. (In Chinese)

[5] T.Jin and Z.J.Zhou, Research and Strategies on the Current situationof Extracurricular Sports Activities in Rural Middle Schools in Anhui Province. Journal of Anhui Normal University(Natural Science). (2007) No. 01. (In Chinese)

[6] X.J.Tang and P.S. Nie,Research and Analysis on the Current situationof Extracurricular Sports Activities in Rural Middle Schools in Hubei Province. Journal of Hubei Sports Science. (2007) No. 03. (In Chinese)

[7] X.P.Shi, Reflection on the Implementation of Course Standard of Physical Culture and Health. Journal of Yunmeng. (2007) No. 5. (In Chinese)

[8] G.C.Lu, Analysis on the Current situationof Physical Activities of Middle School Students. Reform and Opening up. (2011) No. 08. (In Chinese)

[9] J.Y.Su, Research and Analysis on the Current situationof Extracurricular Sports Activities in Rural Middle Schools in Guizhou Province. Journal of Teaching and Management. (2013) No. 24. (In Chinese)

[10]H.H.Wang,Research and Strategies on the Current situationof Extracurricular Sports Activities in Rural Middle Schools in Qingshui County of Gansu Province. Bulletion of Sports Science \& Technoly. (2012) No. 04. (In Chinese)

[11]C. Qin, School Physical Education. Extracurricular Sports Activity. Higher Education Press. 2005.(In Chinese)

[12]L.Ji, School Physical Education and Quality Education. Higher Teacher Education Research. 1999.(In Chinese) 